\title{
Jumping Exercise Restores Stretching-Induced Power Loss in Healthy Adults
}

\author{
Hyun Chul Jung ${ }^{1}$, Nan Hee Lee ${ }^{2}$, Sukho Lee ${ }^{3}$
}

\begin{abstract}
Affiliations: 'University of Louisiana at Monroe, College of Health and Pharmaceutical Sciences, Department of Kinesiology, Louisiana, USA, ${ }^{2}$ Georgia Institute of Technology, College of Sciences, School of Biological Sciences, Atlanta, USA, ${ }^{3}$ Texas A\&M University-San Antonio, College of Education and Human Development, Department of Counseling, Health, and Kinesiology, Texas, USA
\end{abstract}

Correspondence: S. Lee, Texas A\&M University-San Antonio, Faculty for Counseling, Health, and Kinesiology, One University Way Madla 324, San Antonio, Texas 78224, USA. E-mail: slee@tamusa.edu

ABSTRACT The purpose of this study was to examine the acute effects of jumping exercise (JE) immediately after different stretching protocols on flexibility and power in healthy adults. This study was conducted with a balanced crossover design. Thirteen healthy males $(25.4 \pm 3.46$ years old $)$ voluntarily participated in this study. All participants randomly completed four trials, including three different stretching protocols; 1) static stretching (SS), 2) dynamic stretching (DS), 3) proprioceptive neuromuscular facilitation stretching (PNFS), and 4) a non-stretching control (NS) followed by the JE with seven-day intervals between tests. JE was composed of three sets of five tuck jumps. Flexibility was determined by the ability to perform a straight leg raise (SLR) and power by vertical jump performance (VJP). Both SLR and VJP were measured at four time points; 1) baseline, 2) post-jogging, 3) post-stretching, and 4) post-JE; $4 \times 4$ repeated measures analysis of variances were applied. There were significant interaction effects on SLR $(F=8.935, p<.001)$ and VJP $(F=3.965$, $\mathrm{p}=.009)$. The SLR score increased in all stretching protocols except the NS protocol post-stretching and postJE. After stretching, the VJP score decreased in the NS (-2.6\%), SS (-3.6\%), and PNFS (-4.4\%) protocols but maintained a positive score for the DS (1.8\%) protocol. However, the VJP score recovered to the previous value in the SS (3.2\%) and PNFS (6.5\%) protocols after the jumping exercise. The present study suggests that jumping exercise immediately after SS and PNFS protocols could be an efficient program for restoring stretching-induced power loss in healthy adults.

KEY WORDS flexibility, jumping exercise, power, stretching

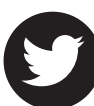

$@$ @JSSMontenegro

POTENTIATING EXERCISE AND POWER

http://mjssm.me/?sekcija=article\&artid=164

\section{Introduction}

The importance of warm-up prior to main exercise and sports events has been widely recognized for preventing injuries and optimizing exercise performance (Woods, Bishop, \& Jones, 2007). The warm-up programme generally consists of light aerobic activity, stretching, and sport-specific movements for 15-20 minutes (Woods et al., 2007). Light aerobic activities, such as jogging and cycling, have been known to increase body temperature and blood circulation, which leads to improved exercise performance, such as flexibility, strength, and power (Bishop, 2003; Young \& Behm, 2002).

Stretching exercises are commonly applied following light intensity aerobic activities to increase flexibility and decrease injuries (Hartig \& Henderson, 1999). Various stretching protocols, such as static stretching (SS), dynamic stretching (DS), and proprioceptive neuromuscular facilitation stretching (PNFS), have been introduced as pre-exercise stretching protocols. SS is a common stretching technique that serves as a warm-up programme, and this technique has been known to improve range of motion and decrease muscle soreness (Andersen, 2005). The DS has become a preferred choice in the athletic community in recent years, because this technique has been shown to improve performances in power (Franco, Signorelli, Trajano, Costa, \& de Oliveira, 2012), sprints (Fletcher \& Jones, 2004), and strength (Sekir, Arabaci, Akova, \& Kadagan, 2010) despite musculotendinous unit (MTU) stiffness being decreased (Herda et al., 2013). The PNFS is widely

Received: March 132018 | Accepted after revision: June 022018 | First published online: September 012018

(C) 2018 by the author(s). License MSA, Podgorica, Montenegro. This article is an open access article distributed under the terms and conditions of the Creative Commons Attribution (CC BY).

Conflict of interest: None declared. 
applied in a clinical environment to enhance both active and passive ranges of motion with the ultimate goal being to optimize muscular performance (Bradley, Olsen, \& Portas, 2007). The PNFS protocol is not commonly recommended immediately prior to explosive athletic movement because it could diminish jump performance and muscle strength (Bradley et al., 2007; Marek, Cramer, Fincher, \& Massey, 2005). However, this protocol provides great benefits for those who participate in exercises that require great flexibility, such as gymnastics. Many studies on stretching and exercise performance have been conducted, but the outcomes are still controversial depending on the duration and type of stretching protocols (Bradley et al., 2007; Fletcher \& Jones, 2004; Franco et al., 2012; Marek et al., 2005; Sekir et al., 2010), the performer's baseline status (Behm \& Chaouachi, 2011; Donti, Tsolakis, \& Bogdanis, 2014), and gender differences (Donti et al., 2014).

As a final component of warm-up programmes, potentiating exercise is applied with specific forms related to upcoming sports events or activities. The potentiating exercise focuses on the intensity of activities that include various explosive movements such as sprinting, jumping, and throwing (Till \& Cooke, 2009; Tillin \& Bishop, 2009). It has been known to facilitate a high degree of central nervous stimulation; thus, the recruitments of fast twitch motor units are enhanced (Hamada, Sale, MacDougall, \& Tarnopolsky, 2000; Hodgson, Docherty, \& Robbins, 2005). In particular, plyometric type jumping exercise (JE) is often used as a form of potentiating exercise. However, it is not well understood how plyometric jumping exercise influences flexibility and power performance when combined with different stretching protocols. A previous study reported that three sets of five jumping exercises after static stretching restored counter-movement jump (CMJ) in international fencing athletes (Tsolakis, Bogdanis, 2012). However, Donti et al. (2014) reported that one set of five tuck jumps did not improve power performance in elite gymnasts. Another study also reported that jumping exercise immediately after DS did not improve vertical jump performance (Turki et al., 2011).

As mentioned previously, the performance outcomes, including strength and power, following different stretching are well understood in the previous studies (Behm \& Chaouachi, 2011; Bishop, 2003), but it is unclear the how the jumping exercise affects performance when combined with different types of stretching (i.e., SS, DS, and PNFS) even though warm-up programmes commonly include stretching and explosive movements. In this study, we have specifically selected a jumping exercise (tuck jump) as a potentiating exercise because strength and power in lower limbs play an important role in most sports events. In addition, the tuck jump does not require specific techniques, which enable it to be applied for general populations, such as healthy adults. Thus far, most studies regarding stretching and performance predominantly have used athletes as subjects (Donti et al., 2014; Tsolakis \& Bogdanis, 2012; Turki et al., 2011). However, it is important to know that athletes are a unique group in comparison to the general population, because their body (i.e., physiological, functional) and mind (psychological) respond differently to exercise or warm-up (Dehkordi, 2001; Koch et al., 2003). We believe the importance of warm-up programmes should be emphasised in healthy adults as well as athletes as the number of participants who exercise has increased among healthy adults. This study would provide practical information to healthy adults, which enable the application of a warm-up programme before exercise or a sport event. Therefore, the purpose of this study was to examine the acute effects of jumping exercise immediately after different stretching protocols on flexibility and power in healthy adults. We hypothesize that jumping exercise immediately after different stretching protocols will enhance flexibility and power performance in healthy adults.

\section{Methods}

\section{Participants}

Participants were recruited through advertisements at the university. Seventeen healthy collegiate males voluntarily participated in this study. No subjects engaged in any stretching-related exercise (i.e. yoga, Pilates), and had no skeletomuscular injuries in the previous two to three years. During the study period, four subjects dropped out due to personal reasons. Therefore, thirteen healthy males $(25.4 \pm 3.46$ years, $171.7 \pm 6.97 \mathrm{~cm}$, $77.0 \pm 12.28 \mathrm{~kg}$ ) completed the study. The study procedures, including the potential risk factors, were explained to the participants. Written informed consent was obtained from the participants prior to testing. This study was approved by the Institutional Review Board of Texas A\&M University-San Antonio.

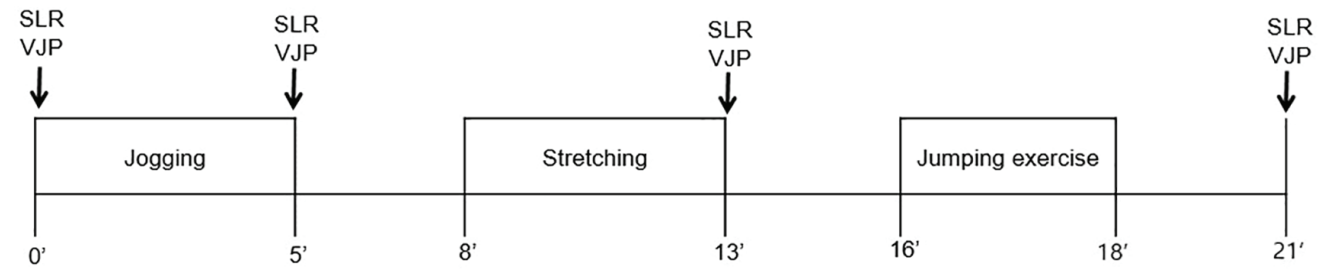

${ }^{*}$ SLR; straight leg raise, VJP; vertical jump performance

* Stretching protocols: three different stretching protocols (SS, DS, PNFS) and NS control were applied in a 7-day interval

* Jumping exercise: three sets of five times tuck jump

FIGURE 1 Study procedure

Note. * SLR; straight leg raise, VJP; vertical jump performance; * Stretching protocols: three different stretching protocols (SS, DS, PNFS) and NS control were applied in 7-day intervals; ${ }^{*}$ Potentiating exercise: three sets of five tuck jumps. 


\section{Study design}

This study was conducted in a balanced crossover design (Figure 1). Height and weight were measured via the use of a wall-mounted stadiometer (Stadi-O-Meter ${ }^{\circ}$, Rockton, USA) and a digital scale (SECA, Hamburg, Germany), respectively. Prior to stretching, participants performed five minutes of jogging on a treadmill $(6.4 \mathrm{~km} /$ hour$)$. Three different stretching protocols (SS, DS, and PNFS) combined with JE were randomly applied with a seven-day interval between tests. Non-stretching combined with JE served as the control group. Straight leg raise (SLR) and VJP were measured at four time points; baseline, post-jogging, post-stretching, and post-JE with three minutes of recovery time between the measurements.

\section{Stretching protocols}

Three stretching (SS, DS, and PNFS) protocols were specially targeted towards lower limb muscles (calf, hamstrings, quadriceps, and gluteus maximus). Each stretching protocol was applied to a single muscle group of 30 seconds with mild discomfort for a total of five minutes. For NS control, participants sat in a chair for five minutes. The stretching protocols were modified based on previous studies (Donti et al., 2014; Franco et al., 2012). The components of stretching techniques are described in Table 1.

\section{TABLE 1 Stretching techniques}

\begin{tabular}{|c|c|c|}
\hline Stretching & Target & Description \\
\hline \multirow{4}{*}{ Static } & Calf & $\begin{array}{l}\text { Subjects stood facing a wall further than arms-length away. Next, subjects leaned into the wall and placed their hands on } \\
\text { the wall for stability. Subjects placed their right foot forward while leaving their left foot back. While leaning into the wall, } \\
\text { subjects' calf muscles are stretched until the point of slight discomfort. Position was repeated for the opposite side. }\end{array}$ \\
\hline & Hamstring & $\begin{array}{l}\text { Subjects sat on a mat with left leg fully extended. Next, the right leg flexed at the knee and the foot was placed alongside } \\
\text { the medial aspect of the knee of the left leg. The subjects then bent forward, keeping the back straight, while grabbing the } \\
\text { dorsiflexed left foot until the hamstring stretched to the point of slight discomfort. Position was repeated for the opposite } \\
\text { side. }\end{array}$ \\
\hline & Quadriceps & $\begin{array}{l}\text { Subjects stood facing a wall, placing their left hand on the wall for stability. The right knee was flexed so that the right } \\
\text { hand could hold the right ankle and pull toward to hip until slight discomfort in the quadriceps. Position is repeated for the } \\
\text { opposite side. }\end{array}$ \\
\hline & $\begin{array}{l}\text { Gluteus } \\
\text { maximus }\end{array}$ & $\begin{array}{l}\text { Subjects stood facing a wall and flexed the right hip. Next, the knee was flexed so that the subject could hold the right } \\
\text { ankle. While holding the right ankle, the knee was pulled towards the chest until slight discomfort in the glutes. Position is } \\
\text { repeated for the opposite side. }\end{array}$ \\
\hline \multirow{4}{*}{ Dynamic } & Calf & $\begin{array}{l}\text { Walking with dorsiflexion to plantar flexion: Subjects stood and raised one foot with the knee fully extended. Then, subjects } \\
\text { dorsiflex the ankle joint intentionally so that the toe was pointing upward. Then, subjects plantar flex the ankle joint } \\
\text { intentionally so that the toe was pointing downward. Both positions of flexion should cause slight discomfort. }\end{array}$ \\
\hline & Hamstring & $\begin{array}{l}\text { Frankenstein walks: Subjects flexed at the hip while intentionally keeping the knee fully extended. Leg was then flexed at } \\
\text { the hip as high as possible until slight discomfort was felt in the hamstring. } \\
\text { Inch worms: Subjects started in the push-up position. Keeping the knees extended, subjects walked feet forward towards } \\
\text { their hands. Once slight discomfort is felt, subjects walked hands forward, keeping knees extended, back to start position. }\end{array}$ \\
\hline & Quadriceps & $\begin{array}{l}\text { Heel-ups: subject kicked heels towards buttocks while moving forward } \\
\text { High knee up to chest: subject running forward with high knee up }\end{array}$ \\
\hline & $\begin{array}{l}\text { Gluteus } \\
\text { maximus }\end{array}$ & $\begin{array}{l}\text { Walking lunges: Subjects took one large step forward with either the right or left foot. Next, with both arms out in front, the } \\
\text { subject rotated their upper bodies, keeping arms horizontal to the ground. }\end{array}$ \\
\hline \multirow{4}{*}{ PNF } & Calf & $\begin{array}{l}\text { Subjects laid in the supine position with knees fully extended. Next, both legs were flexed at the hip until perpendicular to } \\
\text { the floor. This dorsiflexed both feet at the ankle joints so that the instructor could place both hands on the subject's feet. } \\
\text { First, the subject pushed against the instructor's force for } 10 \text { sec. (contraction phase) and then relaxed for } 20 \text { sec. (relaxation } \\
\text { phase). Instructor then pushed the subject's feet (extreme dorsiflexion) during relax phase. }\end{array}$ \\
\hline & Hamstring & $\begin{array}{l}\text { Subjects laid in the supine position with both knees fully extended. Next, one foot was placed on the instructor's shoulder } \\
\text { and flexed the hip joint. First, the subject pushed against the instructor's force for } 10 \mathrm{sec} \text {. (contraction phase), and then } \\
\text { relaxed for } 20 \text { sec. (relaxation phase). Instructor then pushed the leg forward (extreme hip flexion) pushing the subject's } \\
\text { knee toward the chest during relaxation phase. }\end{array}$ \\
\hline & Quadriceps & $\begin{array}{l}\text { Subjects laid in the prone position on the mat with knees fully extended. Next, one knee was flexed until perpendicular to } \\
\text { the floor. Instructor then placed their hand on the subject's ankle. First, subjects pushed against the instructor's force for } \\
10 \text { sec. (contraction phase), and then relaxed for } 20 \text { sec. (relaxation phase). Instructor then pushed the foot towards the hip } \\
\text { (extreme flexion of the knee) during the relaxation phase. }\end{array}$ \\
\hline & $\begin{array}{l}\text { Gluteus } \\
\text { maximus }\end{array}$ & $\begin{array}{l}\text { Subjects sat on the floor and brought the soles of the feet together. Subjects then brought their heels in towards their } \\
\text { body. Instructor sat with knees behind subject and placed their hands on the subject's knees. First, subjects pushed against } \\
\text { the instructor's force for } 10 \text { sec. (contraction phase), then relaxed for } 20 \text { sec. (relaxation phase). Instructor then pushed the } \\
\text { subject's knees toward to floor (extreme hip abduction) during the relaxation phase. }\end{array}$ \\
\hline
\end{tabular}

\section{Jumping exercise programme}

After each stretching protocol was completed, participants performed three sets of five tuck jumps with 30-second intervals between sets (Donti et al., 2014). 


\section{Exercise performance testing}

SLR and VJP tests were selected for the measurements of flexibility and power performance. The SLR was measured with a goniometer (Baseline stainless steel goniometers, USA). Participants lay in a supine position on a medical bed with their backs flat to prevent possible pelvic rotation. Then participants raised their dominant leg as far as possible while maintaining the knee fully extended with ankle joint in a dorsiflexion position. One lever of the goniometer was marked on the lateral midline of the pelvis, while the pivot was placed on the lateral aspect of the hip joint, at the greater trochanter. The opposite leg was firmly held down to prevent flexion at the hip joint. Participants performed two-trials, and the highest score was recorded. The intra-class correlation coefficient (ICC) for SLR was 0.98. The VJP test was measured as a marker of power performance. Participants first raised their right arm on the measuring bar with a fully extended elbow, which marked an initial point. Then, they were instructed to jump with their maximal effort as high as possible. The jump height was calculated from maximal jump height minus initial point. Each participant performed two trials, and the highest score was recorded. The ICC for VJP was 0.93 .

\section{Statistical analysis}

SPSS (version 24.00, SPSS Inc., Chicago, Illinois) was used for statistical analysis. All data were presented as mean and standard deviations. The percentage change of scores was also calculated for all measures. $4 \times 4$ repeated measures analysis of variances (ANOVA) were applied to analyse the changes of SLR and VJP between (a) different stretching protocols and (b) time sequences. If any significant interactions or main effects were detected, repeated measure ANOVAs with a Bonferroni post hoc test was applied. One-way ANOVAs were applied to analyse the percentage changes of SLR and VJP between the different protocols at the post-jogging, the-post-stretching, and the post-JE. Partial eta squared $\left(\eta_{\mathrm{p}}{ }^{2}\right)$ was used to classify the effect size. The reliability estimated for the best score in SLR and VJP was determined by calculating the intra-class correlation coefficient (ICC) (Wood, \& Zhu, 2006). The level of statistical significance was set at $\mathrm{p}<.05$.

\section{Results}

Flexibility

There was a significant interaction effect (protocol $\times$ time) on the straight leg raise $(\mathrm{F}=8.935, \mathrm{p}<.001$, $\left.\eta_{\mathrm{p}}{ }^{2}=.427\right)$. There were also significant effects for time $\left(\mathrm{F}=61.789, \mathrm{p}<.001, \eta_{\mathrm{p}}{ }^{2}=.837\right)$ and trial $(\mathrm{F}=17.739$, $\left.\mathrm{p}<.001, \eta_{\mathrm{p}}{ }^{2}=.596\right)$. The Bonferroni post hoc test showed that the SLR score significantly increased in all trials after jogging. Although the SLR score increased after SS, DS, and PNFS, this score did not change after NS. Jumping exercise immediately after all stretching protocols did not provide additional benefit in flexibility. Overall, jumping exercise immediately after three stretching protocols increased SLR from baseline. Figure 2 describes the changes in flexibility.

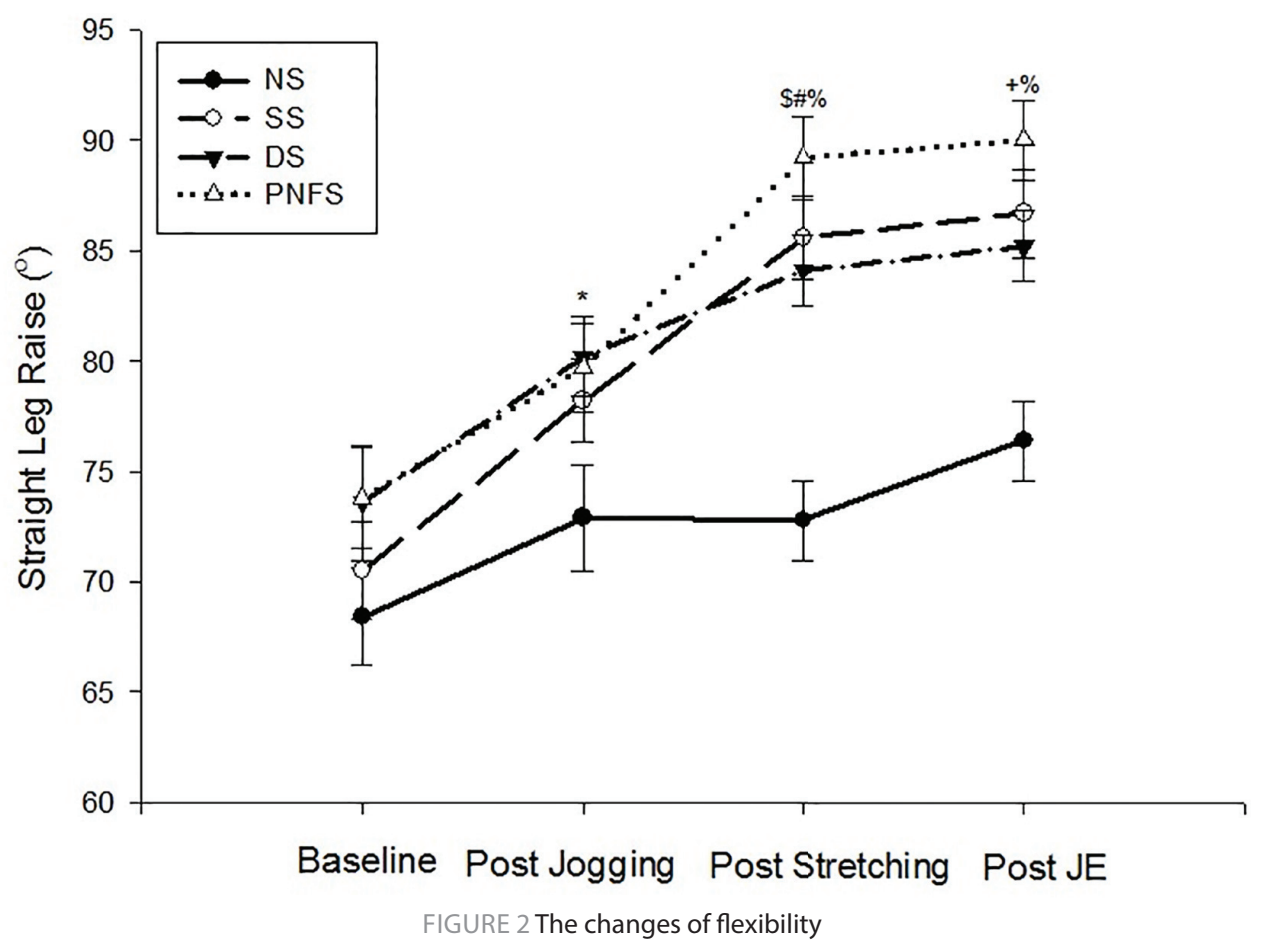

Note. Values are mean and standard deviation; NS: non-stretching; SS: static stretching; DS: dynamic stretching; PNFS: proprioceptive neuromuscular facilitation stretching; JE: jumping exercise; ${ }^{*} \mathrm{p}<.05$, significantly improved from baseline in all protocols; ${ }^{\$} \mathrm{p}<.05$, significantly improved from baseline in all protocols; ${ }^{+} \mathrm{p}<.05$, significantly improved from baseline in all protocols; ${ }^{*} \mathrm{p}<.05$, significantly improved from post-jogging in SS, DS, PNFS; ${ }^{\mathrm{p}}<.05$, significantly differed between SS, DS, PNFS and NS post-stretching and post-JE. 
Power

A significant interaction effect (protocol $\times$ time) on VJP was observed $\left(\mathrm{F}=3.965, \mathrm{p}=.009, \eta_{\mathrm{p}}{ }^{2}=.248\right)$. There was also a significant time effect $\left(\mathrm{F}=20.403, \mathrm{p}<.001, \eta_{\mathrm{p}}{ }^{2}=.630\right)$. The Bonferroni post hoc test revealed that the VJP score significantly increased in all trials after jogging. However, the VJP score decreased after the NS, SS, and PNFS protocols, although it did not change after DS. After the jumping exercise, the VJP score was only restored post-jogging in the SS and PNFS protocols, whereas it did not change in the NS and DS protocols. Overall, the jumping exercise immediately after DS and PNFS significantly improved the VJP from baseline. Figure 3 represents the changes of VJP.

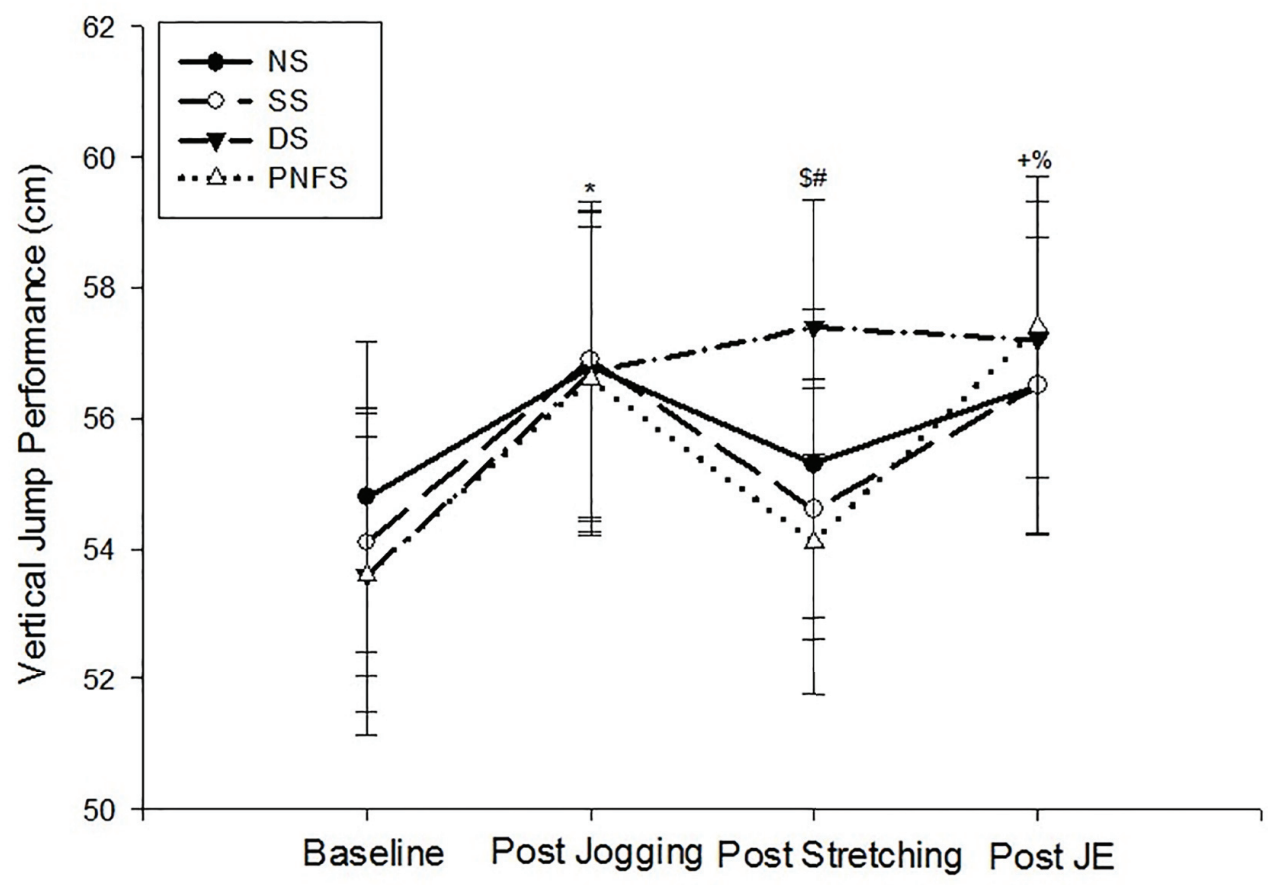

FIGURE 3 The changes of vertical jump performance

Note. Values are mean and standard deviation; NS: non-stretching; SS: static stretching; DS: dynamic stretching; PNFS: proprioceptive neuromuscular facilitation stretching; JE: jumping exercise; ${ }^{*} \mathrm{p}<.05$, significantly improved from baseline in all protocols; ${ }^{5} \mathrm{p}<.05$, significantly improved from baseline only in $\mathrm{DS} ;{ }^{+} \mathrm{p}<.05$, significantly improved from baseline in DS and PNFS; ${ }^{*}<<.05$, significantly decreased from post-jogging in NS, SS, PNFS; ${ }^{\%} \mathrm{p}<.05$, significantly improved from post-stretching in SS and PNFS.

\section{Discussion}

This study was aimed at investigating the acute effects of jumping exercise immediately after different stretching protocols on flexibility and power in healthy adults. The main findings are as follows: 1) jumping exercise immediately after the DS and PNFS protocols improves flexibility and power from the baseline; 2) jumping exercise restores the static and PNF stretching-induced power loss in healthy adults.

The flexibility and power increased in all groups after jogging in the present study. It is known that increasing body temperature through light intensity activities provides physiological benefits (Bishop, 2003; Young \& Behm, 2002). With an increase in body temperature, blood flow increases through vasodilation; thus, more oxygen might be supplied to working muscle as well as increase nerve transmission (Bishop, 2003). Although this study did not directly measure body temperature, we assume that increased body temperature through jogging may improve flexibility and power performance.

In the current study, the percentage increase in SLR from jogging was greater in SS (9.4\%), DS (4.9\%) and PNFS (11.9\%) trials than NS (-0.1\%) trial at post-stretching. Even though there were no statistical differences in SLR among the three stretching protocols, the PNFS protocol showed the greatest improvement in SLR. PNFS is known as the most effective method for increasing range of motion in joints and flexibility (Konrad, Gad, \& Tilp, 2015). This contract-relax stretching method may have an impact on autogenic inhibition, especially the Golgi tendon organ. Increasing tension during the contraction phase may increase antagonist muscle activity while the function of the Golgi tendon organ decreases during the relaxation phase; therefore, the joint range of motion increased (Konrad et al., 2015). The DS protocol showed the lowest improvement in SLR among three stretching protocols. A previous study reported that DS is not as effective in increasing flexibility compared to SS and PNFS (Behm \& Chaouachi, 2011).

In the present study, VJP significantly decreased in the NS (-2.6\%), SS (-3.6\%), PNFS (-4.4\%) trials but did not change in the DS (1.8\%) trial post-stretching. Non-dynamic stretching, such as static and PNF stretching, has been known to reduce power performance (Behm \& Chaouachi, 2011). Wallmann, Mercer, \& McWhorter 
(2005) demonstrated that three sets of 30 seconds static stretching decreased jump performance (-5.6\%). Another study also reported that four reps of 30 seconds SS and PNFS ( $5 \mathrm{sec}$ contract and $25 \mathrm{sec}$ relaxation phase) for five minutes, decreased vertical jump performance (SS; 4\%, PNFS; 5.1\%). The mechanism underlying these results demonstrated that prolonged static stretching might inhibit the neural drive and asynchronies of muscle activity (Power, Behm, Cahill, Carroll, \& Young, 2004). The stretch-induced impairment of the length-tension relationship may be another factor that limits further motor unit recruitments (Costa et al., 2012). However, the VJP score was maintained after the DS trial in the present study. We assume that DS may have a different role in power performance compared with other stretching protocols. DS involves many active movements, which induce physiological changes such as increased heart rate, body temperature, and altering other metabolic factors (Fletcher, 2010). Even though a previous study speculated that MTU stiffness is decreased after DS (Herda et al., 2013), which is similarly shown in other stretching protocols (Ryan et al., 2014), the physiological benefits may outweigh the stretching-induced power loss. This study confirmed that five minutes of static and PNF stretching alone reduce VJP while DS did not affect the improved VJP induced by jogging.

A plyometric-type jumping exercise after stretching is commonly applied as a potentiating exercise to promote muscle activity. In the present study, jumping exercise restored the VJP in the SS (3.2\%) and PNFS (6.5\%) protocols whereas this score did not significantly change in the NS and DS protocols. A previous study reported that the combination warm-up (run+stretch+jumps) programme showed the highest score in jumping performance compared to running alone or a run+stretch warm-up programme (Young \& Behm, 2003). Another study reported that 45 seconds of static stretching decreased CMJ (5.5\%), but 3 sets $\times 5$ tuck jumps immediately after stretching restored the power performance in international fencing athletes (Tsolakis \& Bogdanis, 2012). Donti et al. (2014) conducted different volumes of jumping exercise. The study reported that 3 sets $\times 5$ tuck jumps after 30 sec static stretching enhanced CMJ, but one set of five times tuck jump did not improve CMJ in elite gymnasts.

There are various possible reasons that additional jumping exercise after stretching provides benefit to power performance. The first theory is that various explosive-movements may increase central nerve stimulation, which involves the Hoffmann Reflex (H-reflex), resulting in greater fast twitch motor unit recruitments (Hodgson et al., 2005). The second theory involves phosphorylation, which produces more ATP, resulting in greater muscle activation at the structure level of skeletal muscle (Rixon, Lamont, \& Bemben, 2007). It has also been proposed that explosive movements recruit more fast twitch muscle fibres that lead to improved power performance after JE (Hamada et al., 2000). However, the additional jumping exercises after DS do not provide positive benefits over VJP. Behm, Button, Barbour, Butt, \& Young (2004) pointed out the importance of balance between post-activation potentiation exercise and fatigue. A previous study supported our result that dynamic stretching alone improved muscular performance, but an additional $3 \times 3$ times of tuck jumps did not provide the benefits on VJP (Turki et al., 2011). The current study suggests that jumping exercise (5 times $\times 3$ set) after DS may not provide additional benefit over VJP, while undertaking this programme immediately after SS and PNFS may restore the stretching-induced power loss.

The present study suggests that jumping exercise immediately after SS and PNFS protocols could be an efficient programme for restoring stretching-induced power loss in healthy adults. However, jumping exercise after DS did not provide additional benefit to power performance.

\section{Acknowledgements}

We would like to express our gratitude to the people who supported this study and the participants who joined the programme voluntarily.

\section{REFERENCES}

Andersen, J. C. (2005). Stretching before and after exercise: effect on muscle soreness and injury risk. Journal of Athletic Training, 40(3), 218-220.

Behm, D. G., \& Chaouachi, A. (2011). A review of the acute effects of static and dynamic stretching on performance. European Journal of Applied Physiology, 111(11), 2633-2651. doi: 10.1007/s00421-011-1879-2.

Behm, D. G., Button, D. C., Barbour, G., Butt, J. C., \& Young, W. B. (2004). Conflicting effects of fatigue and potentiation on voluntary force. Journal of Strength and Conditioning Research, 18(2), 365-372. doi:10.1519/R-12982.1

Bishop, D. (2003). Warm up I: potential mechanisms and the effects of passive warm up on exercise performance. Sports Medicine 33(6), 439-454.

Bradley, P. S., Olsen, P. D., \& Portas, M. D. (2007). The effect of static, ballistic, and proprioceptive neuromuscular facilitation stretching on vertical jump performance. Journal of Strength and Conditioning Research, 21:223-226. doi:10.1519/R-21046.1.

Costa, P. B., Ryan, E. D., Herda, T. J., Walter, A. A., Hoge, K. M., \& Cramer, J. T. (2012). Acute effects of passive stretching on the electromechanical delay and evoked twitch properties: a gender comparison. Journal of Applied Biomechanics, 28(6), 645-654. doi: 10.1007/s00421-009-1214-3.

Dehkordi, A. G. (2011). The comparison between athlete females and non-athlete females regarding to gener- 
al health, mental health, and quality of life. Procedia-Social and Behavioral Sciences, 15, 1737-1741.

Donti, O., Tsolakis, C., \& Bogdanis, G. C. (2014). Effects of baseline levels of flexibility and vertical jump ability on performance following different volumes of static stretching and potentiating exercises in elite gymnasts. Journal of Sports Science \& Medicine, 13(1), 105-113.

Fletcher, I. M. (2010). The effect of different dynamic stretch velocities on jump performance. European Journal of Applied Physiology, 109(3), 491-498. doi: 10.1007/s00421-010-1386-x.

Fletcher, I. M., \& Jones, B. (2004). The effect of different warm-up stretch protocols on 20 meter sprint performance in trained rugby union players. Journal of Strength and Conditioning Research, 18(4), 885-888. doi: 10.1519/14493.1.

Franco, B. L., Signorelli, G. R., Trajano, G. S., Costa, P. B., \& de Oliveira, C. G. (2012). Acute effects of three different stretching protocols on the Wingate test performance. Journal of Sports Science \& Medicine, 11(1), 1-7.

Hamada, T., Sale, D. G., MacDougall, J. D., \& Tarnopolsky, M. A. (2000). Postactivation potentiation, fiber type, and twitch contraction time in human knee extensor muscles. Journal of Applied Physiology, 88(6), 2131-2137. doi: 10.1152/jappl.2000.88.6.2131.

Hartig, D. E., \& Henderson, J. M. (1999). Increasing hamstring flexibility decreases lower extremity overuse injuries in military basic trainees. The American Journal of Sports Medicine, 27(2), 173-176. doi: 10.1177/03635465990270021001.

Herda, T. J., Herda, N. D., Costa, P. B., Walter-Herda, A. A., Valdez, A. M., \& Cramer, J. T. (2013). The effects of dynamic stretching on the passive properties of the muscle-tendon unit. Journal of Sports Sciences, 31(5), 479-487. doi: 10.1080/02640414.2012.736632.

Hodgson, M., Docherty, D., \& Robbins, D. (2005). Post-activation potentiation. Sports Medicine, 35(7), 585595.

Hoge, K. M., Ryan, E. D., Costa, P. B., Herda, T. J., Walter, A. A., Stout, J. R., \& Cramer, J. T. (2010). Gender differences in musculotendinous stiffness and range of motion after an acute bout of stretching. Journal of Strength and Conditioning Research, 24(10), 2618-2626. doi: 10.1519/JSC.0b013e3181e73974.

Koch, A. J., O’bryant, H. S., Stone, M. E., Sanborn, K., Proulx, C., Hruby, J., Shannonhouse, E., Boros, R., \& Stone, M. H. (2003). Effect of warm-up on the standing broad jump in trained and untrained men and women. The Journal of Strength \& Conditioning Research, 17(4), 710-714.

Konrad, A., Gad, M., \& Tilp, M. (2015). Effect of PNF stretching training on the properties of human muscle and tendon structures. Scandinavian Journal of Medicine \& Science in Sports, 25(3), 346-355. doi: 10.1111/ sms.12228.

Marek, S. M., Cramer, J. T., Fincher, A. L., \& Massey, L. L. (2005). Acute effects of static and proprioceptive neuromuscular facilitation stretching on muscle strength and power output. Journal of Athletic Training, 40(2), 94-103.

Power, K., Behm, D., Cahill, F., Carroll, M., \& Young, W. (2004). An acute bout of static stretching: effects of force and jumping performance. Medicine and Science in Sports Exercise, 36(8), 1389-1396. doi: 10.1249/01.MSS.0000135775.51937.53.

Rixon, K. P., Lamont, H. S., \& Bemben, M. G. (2007). Influence of type of muscle contraction, gender, and lifting experience on postactivation potentiation performance. Journal of Strength and Conditioning Research, 21(2), 500-505. doi: 10.1519/R-18855.1.

Ryan, E. D., Everett, K. L., Smith, D. B., Pollner, C., Tompson, B. J., Sobolewski, E. J., \& Fiddler, R. E. (2014). Acute effects of different volumes of dynamic stretching on vertical jump performance, flexibility and muscular endurance. Clinical Physiology and Functional Imaging, 34(6), 485-492. doi: 10.1111/cpf.12122.

Sekir, U., Arabaci, R., Akova, B., \& Kadagan, S. M. (2010). Acute effects of static and dynamic stretching on leg flexor and extensor isokinetic strength in elite women athletes. Scandinavian Journal of Medicine \& Science in Sports, 20(2), 268-281. doi: 10.1111/j.1600-0838.2009.00923.x.

Till, K. A., \& Cooke, C. (2009). The effects of postactivation potentiation on sprint and jump performance of male academy soccer players. Journal of Strength and Conditioning Research, 23(7), 1960-1967. doi: 10.1519/JSC.0b013e3181b8666e.

Tillin, M. N. A., \& Bishop, D. (2009). Factors modulating post-activation potentiation and its effect on performance of subsequent explosive activities. Sports Medicine, 39(2), 147-166. doi: 10.2165/00007256200939020-00004.

Tsolakis, C., \& Bogdanis, G. C. (2012). Acute effects of two different warm-up protocols on flexibility and lower limb explosive performance in male and female high level athletes. Journal of Sports Science \& Medicine, 11(4), 669-675.

Turki, O., Chaouachi, A., Drinkwater, E. J., Chtara, M., Chamari, K., Amri, M., \& Behm, D. G. (2011). Ten minutes of dynamic stretching is sufficient to potentiate vertical jump performance characteristics. Journal of Strength and Conditioning Research, 25(9), 2453-2463. doi: 10.1519/JSC.0b013e31822a5a79.

Wallmann, H. W., Mercer, J. A., \& McWhorter, J. W. (2005). Surface electromyographic assessment of the effect of static stretching of the gastrocnemius on vertical jump performance. Journal of Strength and Conditioning Research, 19(3), 684-688. doi: 10.1519/R-15904.1.

Wood, T. M. \& Zhu, W. (2006). Measurement theory and practice in kinesiology. Champaign, IL. Human Kinetics, P.28-52.

Woods, K., Bishop, P., \& Jones, E. (2007). Warm-up and stretching in the prevention of muscular injury. Sports 
Medicine, 37(12), 1089-1099.

Young, W. B., \& Behm, D. G. (2003). Effects of running, static stretching and practice jumps on explosive force production and jumping performance. Journal of Sports Medicine and Physical Fitness, 43(1), 21-27.

Young, W., \& Behm, D. (2002). Should static stretching be used during a warm-up for strength and power activities? Strength and Conditioning Journal, 24(6), 33-37. 